\title{
How Quantum Mechanics and General Relativity Can Be Brought Together
}

\author{
Martin Suda \\ Department of Safety \& Security, Optical Quantum Technologies, AIT Austrian Institute of Technology GmbH, \\ Vienna, Austria \\ Email: martin.suda.fl@ait.ac.at
}

Received 25 February 2016; accepted 25 March 2016; published 28 March 2016

Copyright (C) 2016 by author and Scientific Research Publishing Inc.

This work is licensed under the Creative Commons Attribution International License (CC BY).

http://creativecommons.org/licenses/by/4.0/

(c) (i) Open Access

\begin{abstract}
This paper describes an easy and teaching way how quantum mechanics (QM) and general relativity (GR) can be brought together. The method consists of formulating Schrödinger's equation of a free quantum wave of a massive particle in curved space-time of GR using the Schwarzschild metric. The result is a Schrödinger equation of the particle which is automatically subjected to Newtons's gravitational potential.
\end{abstract}

\section{Keywords}

Quantum Mechanics, Schrödinger Equation, General Relativity, Newton's Gravitational Potential, Curved Space-Time, Schwarzschild Metric, Non-Euclidian Geometry

\section{Introduction}

The problem of synthesis of QM and GR has been the subject of much discussion among physicists in recent years. In this short paper, we try to tackle this question by subjecting the Schrödinger equation of a free quantum wave to the non-Euclidian geometry of space-time developed in the formalism of general relativity.

The motivation to do this is justified by the effort to find an easy and pedagogical way of understanding how the most important physical theories developed in the $20^{\text {th }}$ century, QM and GR, can be brought together in the limit of quantum particles that have extremely small masses compared to cosmological objects.

In doing so, we begin by writing down the well-known non-relativistic Schrödinger equation which describes a quantum particle of mass at rest $m_{0}$ (e.g. a neutron) affected by a radial symmetric potential $V(r)$ [1]:

$$
\left[-\frac{\hbar^{2}}{2 m_{0}} \Delta+V(r)\right] \psi(r, t)=i \hbar \frac{\partial}{\partial t} \psi(r, t) .
$$


$\psi(r, t)$ is the wavefunction depending on position $r$ and time $t, \Delta$ is the Laplace operator and $\hbar$ Planck's constant. In the case of Newton's gravitation, $V(r)$ is written as

$$
V(r)=-\frac{G M m_{0}}{r} \text {. }
$$

$G$ is the constant of gravitation and $M$ the mass which causes gravitation (e.g. mass of Earth).

We investigate stationary solutions of Equation (1) by using the ansatz $\psi(r, t)=\mathrm{e}^{-i \overline{\bar{\omega}} t} \varphi(r)$ and obtain

$$
\left[-\frac{\hbar^{2}}{2 m_{0}} \Delta+V(r)\right] \varphi=\epsilon \varphi
$$

omitting reference to $r$ for function $\varphi . \epsilon=\hbar \bar{\omega}$ is the (negative) binding energy ( $\bar{\omega}$ is the frequency) and can be written as $\epsilon=-\frac{\hbar^{2} \kappa^{2}}{2 m_{0}}$ for a particular momentum $\kappa$ of a particle bounded in the potential $V(r)$.

Equation (3) can be treated in complete analogy to the quantization of electron energies in an hydrogen atom, described in standard textbooks of quantum mechanics [1], to obtain energy states and wave functions of a massive particle bounded in Newton's potential $V(r)$ ([2], Section 3.4.3 therein).

Going back to Equation (3), we initially consider a free quantum wave with $V(r)=0$ and obtain the stationary Schrödinger equation

$$
\Delta \psi=-K^{2} \psi
$$

with plane-wave solution $\psi=\mathrm{e}^{i(K r-\bar{\omega} t)}$ and $\frac{\hbar^{2} K^{2}}{2 m_{0}}=T . T$ is the kinetic energy which is identical to the total energy in this special case where $V(r)=0$. Here, $K$ specifies the momentum of the free particle.

Because of the radial-symmetric potential Equation (2), we switch to spherical coordinates rewriting $\Delta$ for s-waves as

$$
\Delta \psi=\frac{2}{r} \frac{\partial \psi}{\partial r}+\frac{\partial^{2} \psi}{\partial r^{2}}=-K^{2} \psi
$$

with spherical wave solution $\psi=\frac{1}{r} \mathrm{e}^{i(K r-\bar{\omega} t)}$ of a free quantum wave.

\section{Free Quantum Wave in Curved Space-Time of GR}

Now let's switch to the relativistic point of view.

Taking GR into account (e.g. [3]), four dimensions (space and time) have to be considered:

$x^{0}=c t, x^{1}=r, x^{2}=\Theta, x^{3}=\Phi$. Here $c$ is the velocity of light. $r, \Theta, \Phi$ denote the spherical coordinates. In the following, we use the covariant and contravariant notation of GR (lower and upper indices). Therefore, the four coordinates can be merged to $x^{\mu}$, where $\mu=0,1,2,3$.

Now, the following idea is discussed: embedding the QM-formalism of a free wave into space-timeformalism of GR, we can change Equation (5) in complete formal analogy by rephrasing $\Delta \psi=-K^{2} \psi$ into

$$
\Delta^{\prime} \psi=-k_{\mu} k^{\mu} \psi \text {. }
$$

$\Delta^{\prime} \psi=\frac{1}{\sqrt{g}} \frac{\partial}{\partial x^{\mu}}\left(\sqrt{g} \frac{1}{g_{\mu \nu}} \frac{\partial \psi}{\partial x^{v}}\right)$ denotes the Laplace operator of a diagonal metric $g_{\mu v}$ in four dimensions applied to $\psi$ [4]. The quantity $g$ denotes the negative determinant of the metric which is specified below. We will use the so-called Schwarzschild metric (see below).

The right hand side of Equation (6) uses the relativistic momenta [3]

$$
p_{\mu}=\hbar k_{\mu}=\left(-\frac{E}{c}, p\right), \quad p^{\mu}=\hbar k^{\mu}=\left(\frac{E}{c}, p\right)
$$


together with the well-known energy relation $E^{2}=\left(m_{0} c^{2}\right)^{2}+p^{2} c^{2}$. The quantity $E$ denotes Einstein's total energy $m c^{2}$. Moreover, $p=m v=\hbar k$ and $\left(\frac{m}{m_{0}}\right)^{2}=\frac{1}{1-(v / c)^{2}}$. The quantity $m_{0}$ denotes the mass at rest and $v(k)$ the velocity (momentum) vector of the free particle. Using $E=\hbar \omega=m c^{2}$ we can extract

$$
E^{2}=\left(m_{0} c^{2}\right)^{2}+p^{2} c^{2} \rightarrow k^{2}=-\left(\frac{m_{0} c}{\hbar}\right)^{2}+\left(\frac{\omega}{c}\right)^{2} \rightarrow \hbar \omega \approx m_{0} c^{2}+\frac{\hbar^{2} k^{2}}{2 m_{0}}
$$

where $E_{k i n}=\frac{\hbar^{2} k^{2}}{2 m_{0}} \ll m_{0} c^{2}$. From Equation (6) to Equation (8) follows that $\frac{\hbar^{2} k^{2}}{2 m_{0}}$ does not now mean the kinetic energy $T$ of a free particle in a flat space of Euclidian geometry (as in Equation (4)) but denotes the kinetic energy of this particle bounded in the space-time geometry of GR where the gravitational potential $V(r)$ plays a crucial role. We will prove this fact below.

Immediately, one deduces from Equation (7) $k_{\mu} k^{\mu}=-\left(\frac{m_{0} c}{\hbar}\right)^{2}$. In summary, Equation (6) reads

$$
\frac{1}{\sqrt{g}} \frac{\partial}{\partial x^{\mu}}\left(\sqrt{g} \frac{1}{g_{\mu \nu}} \frac{\partial \psi}{\partial x^{v}}\right)=\left(\frac{m_{0} c}{\hbar}\right)^{2} \psi
$$

This equation describing a quantum wave in curved space-time of GR is our starting point for further considerations. This quantum wave is not free anymore because it is affected by the non-Euclidian geometry of spacetime. We will see below that this is equivalent to a quantum wave described by a Schrödinger equation in Euclidian geometry where Newton's gravitational potential is included (see Equation (17)). [3])

As promised above the diagonal metric we use is the so-called inverse spherical Schwarzschild metric (e.g.

$$
\frac{1}{g_{\mu \nu}}=g^{\mu \nu}=\operatorname{diag}\left[-\left(1-\frac{r_{s}}{r}\right)^{-1},\left(1-\frac{r_{s}}{r}\right), \frac{1}{r^{2}}, \frac{1}{r^{2} \sin ^{2}(\theta)}\right], r_{s}=\frac{2 G M}{c^{2}} .
$$

$r_{s}$ is called Schwarzschild radius.

If $r_{s}=0$ one gets the inverse spherical Minkowski metric $\operatorname{diag}\left[-1,1, \frac{1}{r^{2}}, \frac{1}{r^{2} \sin ^{2}(\theta)}\right]$. The square root of the negative determinant of the metric yields $\sqrt{g}=\sqrt{-|g|}=r^{2} \sin (\theta)$. Now Equation (9) can be figured out easily accounting for Einstein's summation convention. One obtains the following partial differential equation:

$$
-\frac{1}{\left(1-\frac{r_{s}}{r}\right)} \frac{1}{c^{2}} \psi_{t t}+\frac{1}{r}\left(2-\frac{r_{s}}{r}\right) \psi_{r}+\left(1-\frac{r_{s}}{r}\right) \psi_{r r}=\left(\frac{m_{0} c}{\hbar}\right)^{2} \psi
$$

The subscripts $t$ and $r$ of the wave function $\psi$ denote partial derivatives of time $t$ and coordinate $r$, respectively. Moreover it is assumed that $\psi=\psi(r, t)$ only.

Initially we would like to mention that in case of $r_{s}=0$ Equation (11) yields the Klein-Gordon-Schrödinger equation [5] $-\frac{1}{c^{2}} \psi_{t t}+\Delta \psi=\left(\frac{m_{0} c}{\hbar}\right)^{2} \psi$.

In order to solve Equation (11) for $r_{s}>0$ we choose the product ansatz $\psi=f(t) \varphi(r)$ and obtain

$$
\frac{1}{c^{2}} \frac{f_{t t}}{f}=\left(1-\frac{r_{s}}{r}\right)\left[\frac{1}{r}\left(2-\frac{r_{s}}{r}\right) \frac{\varphi_{r}}{\varphi}+\left(1-\frac{r_{s}}{r}\right) \frac{\varphi_{r r}}{\varphi}-\left(\frac{m_{0} c}{\hbar}\right)^{2}\right]=-\left(\frac{\omega}{c}\right)^{2} .
$$


The LHS depends only on $t$, the RHS only on $r$. Therefore we can equalize each individual side with $-\left(\frac{\omega}{c}\right)^{2}$ which should be a constant. We sum up the rest energy $m_{0} c^{2}$ and the binding energy $\epsilon$ (where $|\epsilon| \ll m_{0} c^{2}$, $\epsilon<0$ ) yielding the total energy $\hbar \omega$ :

$$
\hbar \omega=m_{0} c^{2}+\epsilon \rightarrow\left(\frac{\omega}{c}\right)^{2} \approx\left(\frac{m_{0} c}{\hbar}\right)^{2}+2 \frac{m_{0} \epsilon}{\hbar^{2}}
$$

From the LHS of Equation (12) we obtain $f=\mathrm{e}^{-i \omega t}$. Hence, the RHS of Equation (12) yields

$$
\frac{1}{r}\left(2-\frac{r_{s}}{r}\right) \varphi_{r}+\left(1-\frac{r_{s}}{r}\right) \varphi_{r r}-\left(\frac{m_{0} c}{\hbar}\right)^{2} \varphi=-\left(\frac{\omega}{c}\right)^{2} \frac{1}{\left(1-\frac{r_{s}}{r}\right)} \varphi .
$$

We consider $\frac{r_{s}}{r} \ll 1$. This can be called "Newtonian approximation". The reason for that can be justified as follows: The Schwarzschild radius of Earth amounts to $r_{s}=8.86 \times 10^{-3} \mathrm{~m}$ according to Equation (10) and the radius of Earth is $r_{E}=6.38 \times 10^{6} \mathrm{~m}$ on average. On obtains $\frac{r_{S}}{r_{E}}=1.4 \times 10^{-9}$. This should be the scope of application of Equation (14) on Earth for $r$ as well. We therefore neglect the terms $\frac{r_{s}}{r}$ on the LHS of Equation (14) as a first approximation and on the RHS $\frac{1}{\left(1-\frac{r_{s}}{r}\right)}$ is excellently approximated through $\left(1+\frac{r_{s}}{r}\right)$. The result of this approach is

$$
\frac{2}{r} \varphi_{r}+\varphi_{r r}-\left(\frac{m_{0} c}{\hbar}\right)^{2} \varphi=-\left(\frac{\omega}{c}\right)^{2}\left(1+\frac{r_{s}}{r}\right) \varphi
$$

which can be rewritten by using Equation (13) and Equation (5) as

$$
\begin{gathered}
\Delta \varphi-\left(\frac{m_{0} c}{\hbar}\right)^{2} \varphi=-\left[\left(\frac{m_{0} c}{\hbar}\right)^{2}+2 \frac{m_{0} \epsilon}{\hbar^{2}}\right]\left(1+\frac{r_{s}}{r}\right) \varphi \\
\rightarrow \Delta \varphi=-2 \frac{m_{0} \epsilon}{\hbar^{2}} \varphi-\frac{r_{s}}{r}\left[\left(\frac{m_{0} c}{\hbar}\right)^{2}+2 \frac{m_{0} \epsilon}{\hbar^{2}}\right] \varphi \\
\rightarrow \Delta \varphi=-2 \frac{m_{0} \epsilon}{\hbar^{2}} \varphi-\frac{r_{s}}{r}\left(\frac{m_{0} c}{\hbar}\right)^{2} \varphi
\end{gathered}
$$

because $|\epsilon| \ll m_{0} c^{2}$. Multiplying each side with the factor $-\frac{\hbar^{2}}{2 m_{0}}$ and using $r_{s}=\frac{2 G M}{c^{2}}$ from Equation (10) leads to

$$
\left[-\frac{\hbar^{2}}{2 m_{0}} \Delta+V(r)\right] \varphi=\epsilon \varphi
$$

where we have moved $V(r)$, Newton's gravitational potential of Equation (2), to the left side. Immediately one recognizes that Equation (17) is identical to Equation (3). This means that we obtained the stationary nonrelativistic Schrödinger equation including Newton's gravitational potential. 


\section{Conclusion}

From the considerations above one can conclude that by embedding the Schrödinger equation of a free quantum wave (which is defined in Euclidian space) into curved space-time of GR (which is defined in non-Euclidian space) we obtain the Schrödinger equation of a quantum wave which is subjected to Newton's gravitational potential. Moreover, it has been shown that Newton's potential energy comes from the Schwarzschild metric of GR. The space-time geometry of GR applied to a free quantum wave causes Newton's gravitational force to appear automatically in the Schrödinger equation. In this sense, QM and GR can be harmonized if the "Newtonian approximation" (defined through the ratio Schwarzschild radius/position coordinate to be much smaller than 1) is taken into consideration and they can be brought together without any difficulty.

\section{Acknowledgements}

I am grateful to M. Faber, F. Laudenbach and F. Hipp for many discussions and I. Glendinning for revising the manuscript.

\section{References}

[1] Cohen-Tannoudji, C., Diu, B. and Laloe, F. (1977) Quantum Mechanics I and II. John Wiley and Sons, New York London Sydney Toronto.

[2] Giese, E., Zeller, W., Kleinert, S., Meister, M., Tammer, V., Roura, A. and Schleich, W. (2015) The Interface of Gravity and Quantum Mechanics Illuminated by Wigner Phase Space. http://arxiv.org/abs/1402.0963

[3] Cheng, T.-P. (2000) Relativity, Gravitation and Cosmology. 2nd Edition, Oxford University Press, Oxford.

[4] Spiegel, M.R. (1999) Vectoranalysis, Schaum's Outline. McGraw-Hill, New York.

[5] Rebhan, E. (2010) Theoretische Physik: Relativistische Quantenmechanik, Quantenfeldtheorie und Elementarteilchentheorie. Spektrum. http://dx.doi.org/10.1007/978-3-8274-2603-1 